Cuadernos de Lingüística Hispánica $n^{\circ} .24$

ISSN 0121-053X • ISSN en línea 2346-1829

Julio-Diciembre 2014, pp. 87-104

\title{
Enseñar a leer en Derecho. Análisis textual de la obra Cartas de batalla, crítica al constitucionalismo colombiano*
}

ILENE ROJAS GARCÍA**

zellene7@gmail.com

Recepción: 29 de marzo de 2014

Aprobación: 26 de mayo de 2014

Cómo citar este artículo: Rojas García, I. (2014). Enseñar a leer en Derecho. Análisis textual de la obra Cartas de batalla, crítica al constitucionalismo colombiano. Cuadernos de Lingïística Hispánica, 24, 87-104. Tunja: Uptc.

* Artículo de investigación científica. Hace parte del proyecto «Alfabetización Académica en la CURN» inscrito, a su vez, en el Proyecto Institucional de Competencias Comunicativas de la Corporación Universitaria Rafael Núñez.

* Licenciada en Español y Literatura, Magíster en Linguística de la Universidad Tecnológica de Pereira. Docente coordinadora del Proyecto Institucional de Competencias Comunicativas de la Corporación Universitaria Rafael Núñez (Cartagena, Colombia); miembro fundador del grupo Estudios del Lenguaje y la Educación (Colciencias), vinculada al grupo Huellas Pedagógicas de la Corporación Universitaria Rafael Núñez. 


\title{
Resumen
}

En el Proyecto Institucional de Competencias Comunicativas de la Corporación Universitaria Rafael Núñez, se adelanta la investigación "Alfabetización académica en la CURN". Este estudio enfatiza en el lenguaje como medio de acceso: al conocimiento, a la comunidad científica particular de cada programa y a la cultura investigativa. El trabajo se desarrolla a partir de experiencias de aula, desde donde el docente de lengua identifica las particularidades discursivas de los textos académicos usados en el programa de Derecho (en una primera instancia), y desde allí determina, tanto las dificultades para su comprensión, como las estrategias didácticas y lingüísticas que pueden desarrollarse para superarlas bajo un enfoque de lectura inferencial. En la experiencia descrita, se aborda el libro Cartas de batalla, una crítica al constitucionalismo colombiano, su lectura interdisciplinar permitió responder a las necesidades de docentes y estudiantes al brindar herramientas prácticas que les permitan aprender a partir del texto escrito, de forma detallada y analítica. En la actualidad estamos revisando textos pertenecientes a otras asignaturas del programa de Derecho, con algunos avances en la Facultad de Medicina.

Palabras clave: alfabetización académica, lectura transversal, inferencia, didáctica de la lengua, análisis textual.

\section{Teaching how to read in Law school A textual analysis of the book Cartas de batalla, una crítica del constitucionalismo colombiano}

\begin{abstract}
"Academic literacy at the CURN" is a research project being carried out as a part of the Institutional Project on Communicative Competences at the Corporación Universitaria Rafael Núñez. This study emphasizes on language as a means of access to knowledge, to each program's specific scientific community, and to a research culture. This work is developed through classroom experiences in which the language teacher identifies some discursive particularities of law school course books and determines, both their difficulty for being understood, and the didactic and linguistic strategies that could be developed to overcome these difficulties, through the focus of
\end{abstract}


inferential reading. The experience described above resorted to an interdisciplinary reading of the book Cartas de batalla, una crítica del constitucionalismo colombiano, to meet the needs of teachers and students, by providing practical tools for detailed and analytical learning through the written text. The research is currently revising further texts belonging to other subjects of the Law program, after a similar process at the Faculty of Medicine.

Key words: Academic literacy, transversal reading, inference, language didactics, textual analysis.

\section{Enseigner à lire en Droit Analyse textuelle de l'œuvre Lettres de bataille, une critique au constitutionalisme colombien}

\section{Résumé}

Dans le Projet Institutionnel de Compétences Communicatives de la Corporation Universitaire Rafael Núñez, on développe la recherche «Alphabétisation académique dans la CURN». Cette étude met en relief le langage en tant que moyen d'accès: à la connaissance, à la communauté scientifique propre à chaque programme et à la culture investigatrice. Le travail se développe à partir des expériences dans la salle de classe, d'où l'enseignant de langue identifie les particularités discursives des textes académiques utilisés dans le programme de Droit (d'abord) ; et partir de là, on détermine, bien les difficultés pour sa compréhension, que les stratégies didactiques et linguistiques qui peuvent se développer pour les surmonter sous une approche de lecture inférentielle. Dans l'expérience décrite, on aborde le livre Lettres de bataille, une critique au constitutionnalisme colombien. Sa lecture interdisciplinaire nous a permis de répondre aux besoins des enseignants et des apprenants au moment de fournir des outils qui leur permettent d'apprendre à partir du texte écrit, de manière détaillée et analytique. Actuellement nous sommes en train de réviser des textes appartenant aux autres cours du programme de Droit, avec quelques avancements dans la faculté de médicine.

Mots clés: alphabétisation académique, lecture transversal, inférence, didactique de la langue, analyse textuel. 
Enseñar a leer en Derecho. Análisis textual de la obra Cartas de batalla, crítica al constitucionalismo colombiano

\section{Ensinar a ler em Direito \\ Análise textual da obra Cartas de batalba, uma crítica ao constitucionalismo colombiano}

\section{Resumo}

No Projeto Institucional de Competências Comunicativas da Corporação Universitária Rafael Núñez, é realizada a pesquisa «Alfabetização acadêmica na CURN». Este estudo enfatiza na linguagem como meio de acesso: ao conhecimento, à comunidade científica particular de cada programa e à cultura de pesquisa. 0 trabalho se desenvolve a partir de experiências de sala de aula, desde onde o docente de língua identifica as particularidades discursivas dos textos acadêmicos usados no programa de Direito (em uma primeira instância), e a partir daí determina, tanto as dificuldades para sua compreensão, como as estratégias didáticas e linguísticas que podem desenvolver-se para superá-las sob um enfoque de leitura inferencial. Na experiência descrita, se aborda o livro Cartas de batalha, uma crítica ao constitucionalismo colombiano, onde sua leitura interdisciplinar permitiu responder às necessidades de docentes e estudantes ao brindar ferramentas práticas que lhes permitam aprender a partir do texto escrito, de forma detalhada e analítica. Na atualidade estamos revisando textos pertencentes a outras disciplinas do programa de direito, com alguns avanços na faculdade de medicina.

Palavras chave: alfabetização acadêmica, leitura transversal, inferência, didática da língua, análise textual. 


\section{Introducción}

Leer y escribir no son habilidades terminadas en la escuela secundaria. Se requiere una formación especializada para comprender y producir los textos propios de cada área del saber (Carlino, 2005, p.13), es decir, la universidad debe brindar un espacio de formación en donde los estudiantes accedan a estrategias metodológicas que les permitan aprender a partir del texto escrito (Narvaja, Di Stéfano y Pereira, 2002, p. 31). Este aspecto cobra especial importancia cuando se observa que un amplio conjunto de contenidos del currículo debe ser estudiado a partir de material escrito y como trabajo independiente.

Bajo esta metodología, se espera que los estudiantes comprendan el contenido de los textos e incluso puedan relacionarlos con otros datos discutidos en las clases presenciales. Sin embargo, los estudiantes no poseen herramientas suficientes para comprender a cabalidad los textos especializados que hacen parte de sus currículos. Estos textos se encuentran drásticamente alejados de los documentos que se estudian durante la educación básica y media, con objetivos, destinatarios, estilos, saberes y requisitos cognitivos distintos (Cisneros, Olave y Rojas, 2013, p. 13). En el mismo sentido, los estudios de alfabetización académica demuestran que los docentes de la disciplina no están capacitados para orientar los procesos de lectura -puesto que su formación se centra en otras áreas del conocimiento-.

Además de ello, las publicaciones sobre lectura y escritura en la educación superior, orientan estos procesos enfatizando en las características generales de los textos, mas no en sus particularidades disciplinares. Suele prestarse mayor relevancia a textos de cultura general, artículos de opinión y ensayos que no poseen temáticas, estructuras, ni regularidades discursivas propias del ámbito académico específico. Así mismo, los cursos de acompañamiento en lectura y escritura ofrecen contenidos generales sobre el dominio del texto, entre los que se cuentan la construcción de párrafos, identificación de la idea central, manejo de signos de puntuación, etc., pero muy pocos estudiantes llegan a transponer las estrategias de lectura que aprenden para la comprensión de un texto de cultura general, al enfrentarse a textos especializados en su área del saber. De modo que, al decir de Bailey y Vardi (1999, citado por Carlino, 2005, p. 23): 
Enseñar a leer en Derecho. Análisis textual de la obra Cartas de batalla, crítica al constitucionalismo colombiano

Son los especialistas de la disciplina los que mejor podrían ayudar con la escritura en el nivel superior, no solo porque están familiarizados con las convenciones de su propia materia (aunque muchas veces sin ser conscientes de ellas) sino porque conocen el contenido difícil que los estudiantes tratan de dominar. [Resaltado añadido].

Es precisamente el inciso aclarativo de Bailey y Vardi la preocupación central de este documento: ¿cómo pueden los docentes de la disciplina orientar procesos de lectura y escritura "sin ser conscientes" de las características discursivas de los textos que ellos encargan para leer? Tal conocimiento es imprescindible para identificar en dónde radican las dificultades de los estudiantes y, desde allí, proponer estrategias de aprendizaje. Se llega entonces a una discusión ya planteada (Carlino, 2013; Narvaja, 2011) acerca de la necesidad del trabajo interdisciplinar entre el docente que domina la ciencia objeto de estudio, y quien posee el conocimiento lingüístico, capaz de proponer estrategias de aprendizaje a partir del texto mismo. No obstante, generar espacios de encuentro entre los docentes, suele ser bastante dispendioso, no solo a nivel de las voluntades de los sujetos, sino además como consecuencia de formas de organización institucional que interfieren en los espacios, tiempos y condiciones contractuales.

La propuesta que se realiza intenta responder a lo planteado por Carlino (2013, p. 372) acerca de la necesidad de realizar "indagaciones de corte lingüístico y etnográfico, que describan y analicen los discursos especializados y las prácticas con textos en cada comunidad disciplinar". En la misma línea, se usan herramientas de la Lingüística textual (Van Dijk, 1983; De Beaugrand y Dressler, 1997) y desde la perspectiva del análisis del discurso (Martínez, 2002; Calsamiglia y Tusón, 2007); con miras a desarrollar la lectura inferencial a la luz de los planteamientos de Cisneros, Olave y Rojas (2013).

Se parte entonces de conocer las particularidades discursivas de los documentos que deben leer los estudiantes en los distintos programas universitarios, relacionarlas con las dificultades de comprensión, y diseñar estrategias didácticas que optimicen los procesos lectores y el aprendizaje de los conceptos a partir del texto escrito. De este modo, tanto el docente como los estudiantes podrán utilizar el reconocimiento del andamiaje escrito para acceder de manera más comprensiva a los documentos de su disciplina. Un primer abordaje de este aspecto es el que se desarrolla a lo largo del presente documento.

\section{Metodología}

La asignatura Derecho Constitucional Colombiano se ubica en tercer semestre de la malla curricular del programa de Derecho. Desde ella se propone la lectura de la obra Cartas de Batalla, una crítica al constitucionalismo colombiano (Valencia, 1987), con el objeto de llevar a los estudiantes a comprender y analizar el concepto de derecho como 
conflicto de intereses, evaluando desde allí algunos aspectos de la historia política del país. Dentro de la experiencia, se abordó la lectura de la primera parte de la obra tanto en la clase de Derecho como en el espacio de la asignatura Competencias Comunicativas II $^{1}$ que pertenece al mismo semestre. Así, se plantea un encuentro interdisciplinar entre los conceptos propios de la ciencia jurídica y las posibilidades de comprensión que se abren desde las ciencias del lenguaje.

La experiencia fue realizada en tres fases. La primera fue de tipo exploratorio para observar la dinámica de trabajo en la clase de Derecho, allí se evidenció la lectura fragmentaria por párrafos donde el profesor solicitaba identificar la idea central para luego ampliar la información al respecto; se hizo evidente el parafraseo y la comprensión literal del texto. Por ello, en la segunda fase, la docente de Competencias Comunicativas orientó la lectura para señalar ciertas particularidades discursivas que permitían profundizar en la intención del autor, y con ella, en el conocimiento del tema. Se trataba de compartir con los estudiantes las estrategias usadas por la docente desde la Linguística, que le permitieron comprender el texto en profundidad, a pesar de no tener conocimientos suficientes sobre las ciencias jurídicas, para que ellos pudiesen replicarlas en la lectura de otros documentos de su disciplina.

Durante esta misma fase se buscó generar autonomía en el análisis del texto, de modo que se preparó un taller donde los estudiantes iban siendo guiados conforme avanzaban en la lectura del documento. En la última fase se realizó la evaluación del proceso a nivel metodológico y de saberes.

En el presente documento se abordará la fase de la intervención correspondiente a la lectura guiada por la docente de lengua; se describe el análisis del primer capítulo de la obra, resaltando los elementos textuales que determinan su comprensión. Durante la experiencia, se realizó la lectura lineal del texto, haciendo pausas para comentar los fragmentos de difícil comprensión y generar estrategias de apoyo; por motivos de adecuación discursiva, esta metodología será obviada en este documento para agrupar las estrategias, no en el orden de aparición del documento, sino según sus categorías.

\section{Resultados}

La primera parte de la obra se titula "La gramática de la guerra", aquí se propone un ejercicio de inferencia para determinar la acepción correspondiente del término "gramática"

\footnotetext{
1 En la Corporación Universitaria Rafael Núñez se ha propuesto, dentro de los programas académicos, una asignatura que busca acompañar los ejercicios de lectura y escritura de los estudiantes, en la actualidad existe de manera independiente a los textos específicos de la disciplina, pero se está trabajando para relacionar esta asignatura directamente con los procesos de enseñanza-aprendizaje específicos de cada programa; esta experiencia es un avance en ese sentido.
} 
a partir de los capítulos que componen esta primera parte: "El derecho como contrato social", "El derecho como guerra ritual" y "El constitucionalismo en Colombia". Revisar la relación entre los subtítulos permite anticipar el contenido del texto y comprender las conclusiones a las que quiere llegar el autor. En este sentido, los subtítulos presentan primero un concepto de derecho que habrá de ser controvertido y usado luego para revisar la realidad nacional; el término gramática hace referencia entonces a un ordenamiento interno que es analizado durante esta primera parte de la obra.

Con estos aspectos aclarados, se pasa a la lectura del primer capítulo, en ella emerge una regularidad de los documentos académicos; se trata del estilo rico en términos científicos, construcciones oracionales compuestas y retóricas que le restan claridad al texto y dificultan su comprensión. Por ello, para este caso, se plantea una serie de estrategias orientadas a facilitar el acceso a la información: esquemas, idea central, marcadores discursivos, títulos y elementos retóricos.

\subsection{Elaboración de esquemas}

El ejercicio de lectura de la obra en mención, exige un nivel de dificultad semejante tanto para los estudiantes como para el docente de lengua en tanto que ninguno de ellos posee los preconceptos suficientes a nivel temático, ni la familiaridad con el estilo discursivo propio de la ciencia jurídica. Por ello, el primer párrafo del documento es quizá uno de los que ofrecen mayor nivel de complejidad; para comprenderlo, es menester usar estrategias de lectura dominadas por el docente de lengua, que serán enseñadas también a los estudiantes durante la experiencia.

El constitucionalismo es la provincia de las constituciones, esos mapas políticos que describen el territorio y las relaciones de los individuos y los grupos que luchan entre sí por el reconocimiento y por la supremacía. Tal región es lo que llamamos Estado o régimen político. Y su realidad cotidiana es poder, poderes, relaciones de fuerza, estrategias y tácticas de interpretación y manipulación de los intereses sociales, que compiten entre sí y constituyen un conflicto regulado, una batalla pactada. De ahí el propósito del constitucionalismo: administrar la polémica materia de la vida cotidiana a fin de imponerle un orden racional, un esquema normativo que despliega principios y procedimientos de asignación de recursos y de solución de conflictos a través de los múltiples niveles y sectores de la sociedad (Valencia, 1987, p. 19).

Para facilitar la comprensión de fragmentos como el anterior, es útil identificar los términos clave y relacionarlos, haciendo evidentes los procesos de causa y consecuencia explicados en el texto. Se esquematiza entonces la información en dos momentos: el primero, diseñado por el docente en la preparación de la lectura para comprenderla, y posteriormente 
reelaborado frente a los estudiantes para mostrarles la forma como se va realizando el esquema a partir de la identificación de los conceptos clave y sus relaciones.

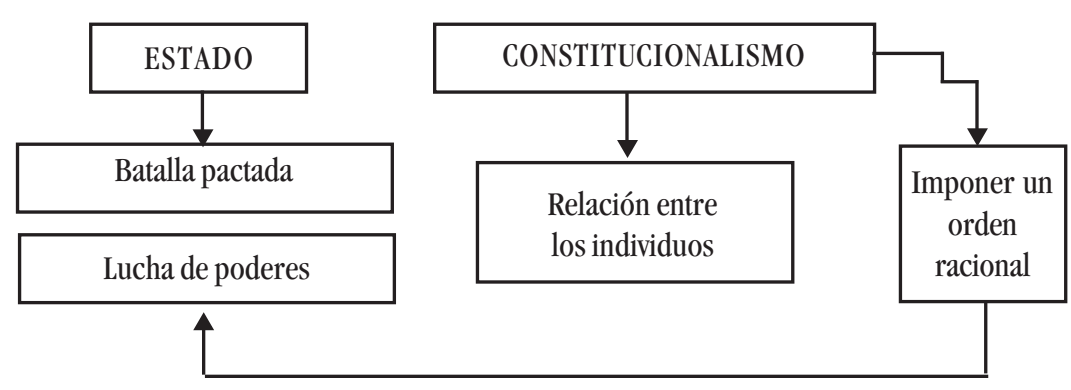

Esquema 1 - Diagrama párrafo 1 - El constitucionalismo

Una vez elaborado el esquema, se lee nuevamente tanto para evidenciar su correspondencia con el texto original, como para memorizar su contenido. Esto último es de especial importancia en tanto que las ideas se van hilando a lo largo del texto y es menester recordar lo ya dicho para comprender la información nueva.

Al diagrama inicial se pueden ir agregando las ideas que vayan surgiendo durante la lectura, este proceso hará consciente al estudiante de las relaciones entre los diversos postulados del autor y evitará dificultades en la comprensión.

Si bien son útiles los organizadores gráficos que se enseñan en la educación media y en los cursos remediales de la universidad, estos no son formatos obligados, sino más bien referentes que permiten poner en vista panorámica los conceptos clave del texto y sus relaciones. Lo que interesa entonces no es el tipo de diagrama, sino las posibilidades que brinda para la comprensión; por ello, de un mismo fragmento pueden aparecer distintos diagramas y la forma de comprobar su adecuación es la correspondencia con el texto, mas no con la forma de un modelo preestablecido.

\subsection{Marcadores discursivos}

El primer párrafo del capítulo posee una estructura expositiva donde se denota la relación del texto con el título: El derecho como contrato social. Siguiendo el diagrama, esto significa que el constitucionalismo impone un orden a la lucha de poderes que caracterizan al Estado. Esta explicación es solo el preámbulo para introducir la postura crítica del autor; para identificarla, se hace necesario enfatizar en los modalizadores discursivos presentes en el fragmento: 
Enseñar a leer en Derecho. Análisis textual de la obra Cartas de batalla, crítica al constitucionalismo colombiano

Las cartas constitucionales o leyes fundamentales bien pueden ser obras de la razón y su lenguaje aparece con frecuencia como el ejemplo arquetípico del discurso racional que describe las cosas como deben ser y no como son. Pero su dinamismo central, su energía es el conflicto civil, la batalla social mucho más que el contrato social. Y el derecho mismo, a causa de su textura abierta, debe ser visto en lo sucesivo dentro de una perspectiva estratégica opuesta a los enfoques contractualistas y funcionalistas aún dominantes en el reino de la jurística. De lo contrario, no es posible dar cuenta del carácter complejo heterogéneo, rebelde, de las relaciones sociales que el derecho pretende concertar. Tan sólo mediante una nueva concepción del derecho y del constitucionalismo, que se abre paso ya en el ancho mundo de las ciencias humanas, parece factible captar la naturaleza intrínsecamente estratégica, es decir, posicional y relacional, de lo social (Valencia, 1987, p.19).

Aparecen entonces tres tipos de elementos lingüísticos que van a evidenciar la posición crítica del autor. Se inicia con un conector de oposición: "pero", que establece un contraste entre dos ideas: 1. La razón como el discurso de las constituciones, y 2. Lo irracional como la esencia misma de las leyes. Posteriormente se incluye un modalizador deóntico que introduce la propuesta del autor, esto es, estudiar el derecho desde una nueva perspectiva; tal perspectiva va acompañada del término "opuesto" como adjetivo que cuestiona las existentes hasta el momento. Termina con un modalizador polémico: "de lo contrario", que anticipa las consecuencias nefastas de continuar el estudio del Derecho según los enfoques "contraactualistas y funcionalistas". Con este análisis, es imposible afirmar el carácter meramente expositivo del texto y ubicar la idea central al inicio del párrafo. Claramente, se ha introducido una polémica bajo la cual la posición del autor, manifiesta en el "debe ser", consiste en orientar el estudio del Derecho a partir de una perspectiva estratégica de los conflictos sociales.

\subsection{Título}

El libro se titula Cartas de batalla: el término carta aparece en el segundo párrafo: "Las cartas constitucionales o leyes fundamentales"; al adicionarles el calificativo "de batalla", se evidencia la propuesta del autor. Así mismo, el primer capítulo ha sido titulado "El derecho como contrato social", de donde cabe analizar dos aspectos: el primero relacionado con la oposición que se encuentra entre este concepto y la idea central del párrafo, podría pensarse que se trata de una ironía, puesto que el autor concibe el derecho como batalla; sin embargo, a lo largo del capítulo, se exponen las corrientes teóricas que han asumido esta postura, de modo que el lector reconozca la necesidad de orientar el estudio del derecho hacia una nueva perspectiva. 


\subsection{Elementos retóricos}

Al tratarse de un texto especializado de las ciencias jurídicas, es claro que contiene elementos usados con una finalidad estética que pueden ocasionar dificultades para la comprensión. Reconocer el sentido de estos términos o formas de decir, depende de la competencia enciclopédica del estudiante, pero también de su capacidad para inferir el sentido a partir de las pistas textuales. Así por ejemplo, el autor propone una alegoría para insertar el tema del orden social como hilo conductor de las teorías del derecho:

Y si hay un hilo conductor, hilo de Ariadna, en el laberinto que constituyen las teorías del derecho formuladas a lo largo de la historia occidental es precisamente la noción de lo legal como un ordenador y nivelador de lo social, lo cual por su parte se percibe siempre como desordenado y desequilibrado (Valencia, 1987, p. 20).

Durante la experiencia se encontró que la mayoría de los estudiantes desconocen el mito de Ariadna, de modo que les era imposible comprender la metáfora propuesta por el autor. Aunque tal conocimiento no resulta relevante para captar el sentido del texto, sí puede permitir una lectura más profunda.

Otro ejemplo de este aspecto se evidencia en el siguiente fragmento: "[...] ese derecho igualitario, nivelador, democrático en su origen, pero antidemocrático en sus métodos y en su finalidad, es la voluntad general en acción, la soberanía nacional convertida en Estado al cual han de someterse tirios y troyanos" (Valencia, 1987, p. 21).

Se hace referencia a los "tirios y troyanos" como metáfora de que todos los pueblos han de someterse a las normas fijadas por el Estado; así como sucede con el "hilo de Ariadna”, el conocimiento de que los tirios y troyanos fueron pueblos en disputa permanente, permite afianzar la idea de la lucha social que se pacifica al subyugarse a las normas del Derecho. Sin embargo, en caso de no tener esta información, es posible deducir simplemente su alusión a la generalidad de las naciones.

Cabe resaltar que algunos conceptos sí son de obligatorio conocimiento, pues determinan las ideas clave del autor. Sucede, por ejemplo, con los autores del Renacimiento que usa Valencia para defender su posición; estos son mencionados en un primer párrafo introductorio para luego ampliar los conceptos haciendo uso de la figura retórica en cuestión:

Conviene aludir a dos elaboraciones teóricas de esta época, que se apartan de la tradición pacifista que venimos reseñando y al mismo tiempo constituyen la formulación inaugural de la tradición estratégica que reivindica este trabajo. Se trata del realismo maquiaveliano, que no maquiavélico, y del pesimismo hobbesiano (Valencia, 1987, p. 21). 
Enseñar a leer en Derecho. Análisis textual de la obra Cartas de batalla, crítica al constitucionalismo colombiano

Valencia, amplía los postulados de Maquiavelo y Hobbes, refiriéndose a ellos como "el secretario florentino y el pensador inglés":

En el secretario florentino, de una parte, se advierte la primera tentativa de pasar del deber ser al ser en la reflexión sobre el Estado [...] En el pensador inglés, de otra parte, se halla la primera exposición sistemática de la teoría del contrato social como sustentación de un proyecto de república autoritaria (Valencia, 1987, p. 21).

Nótese que en el texto no aparece una explicación directa acerca del personaje al que se refiere en cada caso, es necesario buscar las pistas en el texto. La primera herramienta para inferir es el orden de presentación de los autores; la segunda, más adelante en el texto, la mención de "el autor de Leviatán", es decir, Thomas Hobbes. Casos como este obligan a realizar una inferencia léxica que es la extracción del nombre del autor a partir de la corriente: hobbesiano, maquiaveliano; pero también se requiere la capacidad de relacionar la información en el texto e incluir un saber previo referido al conocimiento de las obras y/o los lugares de origen de los autores mencionados.

\subsection{Términos desconocidos}

Siendo que los textos académicos dialogan con otros saberes, incluyen, no solo figuras retóricas, sino también conceptos teóricos y términos técnicos, algunos de los cuales pueden inferirse del cotexto. Así, en la explicación de las corrientes específicas de la Edad Media, se mencionan conceptos como "mesianismo cristiano" o "sociedad empecatada". Para comprender el primer concepto, solo se requiere usar los presaberes de los estudiantes: se refiere al Mesías del cristianismo y la creencia en una restauración del orden. Con respecto al segundo, se entiende que actúa como calificativo de sociedad que, ya se ha reiterado, es desordenada, caótica, incorregible.

Otros casos de comprensión del significado a partir del cotexto pueden verse en el siguiente fragmento referido a Rousseau:

Como doble heredero del jusnaturalismo y del realismo maquiaveliano y hobbesiano, este pensador "entimemático", según la fórmula de Shopenhauer, que arranca siempre de supuestos tácitos u ocultos, funda la política moderna al recomendar como solución a la crisis moral de la civilización europea un tipo de régimen en el cual las buenas leyes se encargarían por sí solas de restablecer las buenas costumbres y de suscitar las buenas conciencias. [... ] En suma, el proyecto russoniano es la auténtica, la suprema ortodoxia del concepto consensual y pacifista de lo jurídico porque parte de la premisa de que el contrato social es la fuente del derecho (Valencia, 1987, p. 21). 
El primer término desconocido es: "entimemático", explicado en la siguiente oración, es decir, se relaciona con "supuestos tácitos u ocultos" (muchos estudiantes también desconocen el sentido de "tácito", pero la conjunción permite establecer el término "oculto" como sinónimo). Posteriormente aparece ortodoxia con la cual se hace posible generar una definición desde la etimología que potencialice también el aprendizaje autónomo: si ortografía significa escritura correcta y ortodoncia alude a una correcta formación dental, entonces la definición para "ortodoxia" se puede inferir comunicando el significado de "doxo" como opinión. Así mismo el término "consensual" del cual, a partir de su semejanza fonética, se puede inferir la derivación de consenso.

Adicionalmente, dado que Valencia se encuentra haciendo un recorrido histórico por las corrientes de pensamiento que han concebido el derecho como contrato social, ha de mencionar ciertos autores que, en muchos casos, son desconocidos para los estudiantes. La dificultad mayor está relacionada, no con el desconocimiento del nombre, sino del campo de estudio que cada autor representa. Este es el caso del siguiente fragmento: "[...] el deber ser, la intención de modelar lo real a partir de un arquetipo arbitrariamente escogido. Podría objetarse, en una perspectiva kantiana que no puedo rechazar, que esto es lo cualitativamente humano [...]" (Valencia, 1987, p. 21).

El autor retoma la filosofía de Kant como interdiscurso para analizar a profundidad las perspectivas de la filosofía clásica. Para los estudiantes, el término "kantiano" hace clara alusión a las ideas de Kant, pero, solo algunos de ellos conocen sus teorías, de modo que les es difícil comprender el verdadero sentido de la objeción que realiza Valencia "desde la perspectiva kantiana". La explicación que aparece a renglón seguido puede ser suficiente para la comprensión de este fragmento, es decir, que desde la perspectiva de Kant, es el sujeto quien establece las normas para gobernar su vida, de modo que no sería necesario replantear el concepto de derecho como ordenador, en tanto que es el mismo ser humano (perteneciente también a la sociedad caótica) quien organiza su realidad.

\subsection{Latinismos}

Los documentos relacionados con las ciencias jurídicas hacen un uso representativo de latinismos, especialmente al tratar temas filosóficos o históricos. Para el caso de este documento, los latinismos son usados como formas de decir y su sentido puede también inferirse del cotexto. Aparece entonces oraciones como "el quid de la cuestión"; "quid" es un término ajeno al dominio conceptual de muchos estudiantes, pero cuyo significado es fácilmente identificable a partir del cotexto, dado que posteriormente aparece la idea central, es decir, "quid" como esencia, idea clave en la relación derecho-sociedad durante la antigüedad clásica, que es, además, reiterativa con todo lo enunciado por el autor. Dice 
Enseñar a leer en Derecho. Análisis textual de la obra Cartas de batalla, crítica al constitucionalismo colombiano

además que en un "nuevo proyecto de sociedad es condición sine que non la creación de un nuevo derecho". El sentido de sine que non puede ser inferido como inherente, obligada.

\subsection{Deícticos}

Para evitar la redundancia, todo texto emplea deícticos y anáforas, muchos de estos elementos hacen referencia a conceptos cercanos en el texto, pero su ambigüedad semántica puede ocasionar tropiezos en la comprensión. Por ello, vale la pena hacer a los estudiantes conscientes de su existencia y usos, así como de los mecanismos de rastreo textual que pueden usar para determinar su significado.

En la obra de Valencia, reconocer el sentido de los términos anafóricos es crucial para comprender el texto, así puede verse en fragmentos como el siguiente:

Tanto para el rey filósofo [...] cuanto para el ciudadano de la república [...] hay un orden metafísico [...] que difiere del orden social en la medida en que el primero se inspira en ciertos valores superiores como el bien, la verdad, la belleza o la justicia, y el segundo se resiste en principio a incorporar tales principios ordenadores y unificadores porque está hecho de otra sustancia, de una esencia degradada o aleatoria que ha de ser ennoblecida o regulada (Valencia, 1987, p. 20).

Los términos "el primero" y "el segundo" se refieren a información ya expresada, es decir, el orden metafísico y el orden social. En su mayoría, tales términos no poseen una complejidad mayor, pero algunos sí requieren una observación más cuidadosa, como sucede a continuación:

En la Edad Media, la escolástica combina sabiamente el realismo aristotélico, el mesianismo cristiano y el moralismo estoico y produce una filosofía del derecho que si bien critica las legalidades terrenas en nombre de la legalidad celeste 0 legitimidad y defiende por vez primera los derechos del pueblo frente a las autoridades estatales, de todos modos termina reiterando la tesis según la cual la sociedad "empecatada" debe ser redimida por el buen ordenamiento legal, que es aquel inspirado en la revelación divina y promulgado por la gestión eclesial.

El jusnaturalismo del Renacimiento y del Barroco prolonga esta línea de pensamiento con dos postulados fundamentales [...]: el individuo es el centro de la vida social, y la comunidad es una invención humana (Valencia, 1987, p. 21).

Al preguntarse por la "línea de pensamiento" que se prolonga luego de la Edad Media, es claro que se debe revisar el fragmento anterior y, según lo postulado hasta el momento, se puede afirmar que se refiere al "ordenamiento legal inspirado en la revelación divina". No obstante, al continuar con la lectura, aparece el concepto de "individualismo" que no pareciera tener relación con el derecho derivado de una ley superior; se necesita entonces devolverse un poco más en el texto: En la Edad Media se empiezan a defender los 
derechos del pueblo frente a los dictámenes de las autoridades estatales, es decir, se rescatan los derechos del individuo. Esta sí es la "línea de pensamiento" a la que se refiere Valencia: los derechos del individuo fundan un estado soberano, que a su vez, habrá de ser el ordenador de la sociedad.

\subsection{Superestructura del texto}

En el último párrafo, se hará evidente el carácter circular del texto, es decir, Valencia plantea su tesis, realiza un recorrido histórico con el que fundamentará sus argumentos, y retomará la propuesta original en el párrafo de cierre:

[... ] puesto que casi siempre se ha pensado el problema del todo desde arriba, desde la montaña, desde el lugar del rey, resulta lógico que el derecho se conciba como una herramienta todopoderosa del espíritu o del poder para disciplinar, para meter en cintura la insumisa energía humana. Por el contrario, si se intenta enfrentar la cuestión del orden $[\ldots]$ desde abajo, desde la llanura, desde el lugar del ciudadano, quizá se abran nuevas perspectivas de estudio e investigación para la jurística y empecemos a comprender. A comprender cómo funciona en realidad el derecho, cuál es su función social efectiva y cuán separado se halla de la conflictiva materia de que está hecha nuestra convivencia (Valencia, 1987, p. 24).

Nótese que aparece de nuevo el marcador "por el contrario" que introduce la propuesta del autor. Se trata de enfatizar en la necesidad de ver el Derecho desde una nueva perspectiva más cercana a la realidad social, alejada de la teoría abstracta e idealizada del deber ser. Este apartado confirma entonces el carácter argumentativo del texto que había sido anunciado desde los primeros párrafos.

\section{Conclusiones}

Como resultado de esta primera fase de la experiencia, se exponen algunos resultados parciales:

La estrategia responde a las necesidades de los docentes, pues los bajos niveles de lectura entorpecen el desarrollo de los contenidos; asimismo, abordar un texto de la disciplina en otra asignatura ayuda a profundizar en los contenidos. Con respecto a los estudiantes, la estrategia brinda herramientas prácticas que les permiten aprender y debatir a partir del texto escrito, de una manera detallada y analítica; así lo evidencian los comentarios realizados durante y al finalizar la intervención. Del mismo modo, el trabajo interdisciplinar facilita a los estudiantes comprender la dinámica de la lengua escrita que atraviesa todos los procesos de formación; muchos de ellos declaran haber aprendido a usar los conocimientos de la clase de Competencias Comunicativas para la comprensión de textos en otras situaciones. Se considera que este es el logro más importante de esta propuesta. 
Enseñar a leer en Derecho. Análisis textual de la obra Cartas de batalla, crítica al constitucionalismo colombiano

El ejercicio tiene implicaciones de tipo logístico que deben ser sorteadas y analizadas en función de su conveniencia. Como se ha venido exponiendo, la intervención se realizó en un grupo que, de manera simultánea, cursaba las asignaturas Derecho Constitucional y Competencias Comunicativas; sin embargo, solo en una de las sesiones se contó con la participación de ambos docentes. De allí podemos extraer algunas conclusiones relacionadas con las ventajas y posibilidades de trabajar con el mismo texto, población y nivel de estudio, paralelamente entre el docente de lengua y el de la disciplina.

En caso de contar con la alineación de estos factores, se facilitará la asistencia y participación de los estudiantes de modo que los encuentros no interfieran en sus horarios y observen el ejercicio como una actividad relacionada directamente con el currículo (esto porque aún hace falta crear una cultura de leer para profundizar en el saber y no solo para obtener una calificación). De otro lado, se hace posible proponer el análisis exhaustivo del documento a partir de otras actividades relacionadas; al ser un texto de carácter argumentativo que cuestiona algunos conceptos del Derecho, cabe la realización de un ejercicio de escritura desde donde el estudiante pueda tomar postura frente a las opiniones del autor, vinculándolas con su propio conocimiento y desarrollando él mismo actitudes críticas. De igual forma, la posibilidad de lecturas intertextuales con situaciones tanto de las teorías histórico-filosóficas enunciadas por el autor para fundamentar sus argumentos, como de la realidad cotidiana, que permitan aterrizar sus críticas en la aplicación del conocimiento de lo jurídico.

Sin embargo, dado que múltiples causas pueden entorpecer la simultaneidad, es posible trabajar únicamente desde la clase de Competencias Comunicativas, dándole un papel activo a los estudiantes del grupo que mejor dominen el tema y a las consultas web para despejar las dudas conceptuales; de igual forma, proponer actividades que relacionen la vida cotidiana con los dominios de la disciplina de modo que se apliquen los contenidos y las reflexiones del documento en un contexto real. Será posible también familiarizarse de manera más analítica con las estrategias que requieren para desempeñarse de manera eficiente en el discurso científico que han elegido como profesión. En ambos casos, cabe resaltar que el nivel de compromiso de los docentes es fundamental en la propuesta, para alcanzar resultados positivos.

Finalizada la actividad, se interrogó a los estudiantes acerca de sus consideraciones sobre el trabajo realizado. Ellos no consideran esencial que el texto abordado se trabaje de manera simultánea en la clase de competencias y en otras de su especialidad; esto es conveniente, puesto que tal situación no puede garantizarse en la totalidad de los casos por cruces en los horarios, estudiantes que matriculan asignaturas de distintos niveles y la esencia misma del Proyecto de Competencias Comunicativas de incluir dentro de una misma clase estudiantes de diversos programas como espacio de diálogo interdisciplinar. Sin

\section{2}


embargo, sí consideran necesaria la presencia tanto del docente de lengua como del docente de la disciplina, de manera que el primero oriente las estrategias comunicativas, mientras que el segundo haga claridad sobre los contenidos específicos del tema. También algunos solicitan dedicar más tiempo de las asignaturas a este tipo de actividades, pues consideran que responden a sus necesidades de formación.

El ejercicio de lectura propuesto busca ser evaluado por el colectivo docente del programa de Derecho, para validarla a nivel de adecuación con la malla curricular, específicamente en el tercer semestre, según su correspondencia con los saberes previos y posteriores que el estudiante debe abordar en el transcurso de su carrera. Asimismo, proponer otros textos o temáticas relacionadas que potencien la transversalidad del conocimiento adquirido a partir del texto elegido y se encuentren directamente relacionados con los conocimientos de las ciencias jurídicas.

Por tratarse de una primera experiencia, se trabajó únicamente el aspecto referente a la lectura, pero es posible y necesario vincular también actividades de escritura y oralidad que desarrollen en los estudiantes las competencias de dominio de lenguaje en un nivel integral. También es necesario incluir este tipo de actividades en los demás programas de la universidad; al respecto, en la actualidad se tiene proyectado con las facultades de Trabajo social, Enfermería y Pedagogía.

Finalmente, dado que desde la visión institucional se propende hacia la solidez científica de su comunidad académica, desde cada facultad los estudiantes desarrollan propuestas de investigación relacionadas directamente con su área de conocimiento, y siendo la lectura y la escritura la base también de este proceso de construcción del conocimiento científico, se requiere vincular estas experiencias con la investigación formativa de los estudiantes, a través de la apertura de una línea de trabajo centrada en la alfabetización académica en las disciplinas, área donde la investigación se encuentra muy activa, especialmente en Australia, Norteamérica y Latinoamérica, y en la cual consideramos que se pueden realizar aportes teóricos valiosos desde nuestros contextos particulares de acción y formación. 
Enseñar a leer en Derecho. Análisis textual de la obra Cartas de batalla, crítica al constitucionalismo colombiano

\section{Referencias bibliográficas}

Calsamiglia, H. y Tusón, A. (1999). Las cosas del decir. Madrid: Ariel.

Carlino, P. (2005). Escribir, leer y aprender en la universidad. Argentina: Fondo de Cultura Económica.

Carlino, P. (2013). Alfabetización académica diez años después. En: Revista Mexicana de Investigación Educativa, 18 (57), pp. 355-381.

Cisneros, M., Olave, G. y Rojas, I. (2013). Alfabetización académica y lectura inferencial. Bogotá: Ecoe.

De Beaugrand, R. y Dressler, W. (1997). Introducción a la Lingüística del texto. Barcelona: Ariel.

Martínez, M.C. (2002). Estrategias de lectura y escritura de textos académicos. Cali: Universidad del Valle.

Narvaja, E. (2011), "La defensa de tesis: rituales y discursividades", conferencia plenaria en el VI Congreso Internacional de la Cátedra UNESCO para el Mejoramiento de la Calidad y Equidad de la Educación en América Latina con Base en la Lectura y la Escritura, Universidad del Norte, Barranquilla, Colombia, 14-17 de junio de 2001.

Narvaja, E., Di Stefano, M. y Pereira, C. (2004). Lectura y escritura en la universidad. Buenos Aires: Universidad de Buenos Aires.

Van Dijk, T. (1983). La ciencia del texto. Buenos Aires: Paidós.

104 